\title{
Research Paper \\ Comparing Negative Emotional Components in Older People With Normal and Poor Quality of Sleep
}

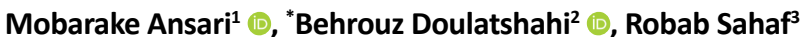

1. Department of Clinical Psychology, University of Social Welfare and Rehabilitation Sciences, Tehran, Iran.

2. Substance Abuse and Dependence Research Center, Department of Clinical Psychology, University of Social Welfare and Rehabilitation Sciences, Tehran, Iran.

3. Department of Gerontology, University of Social Welfare and Rehabilitation Sciences, Tehran, Iran.

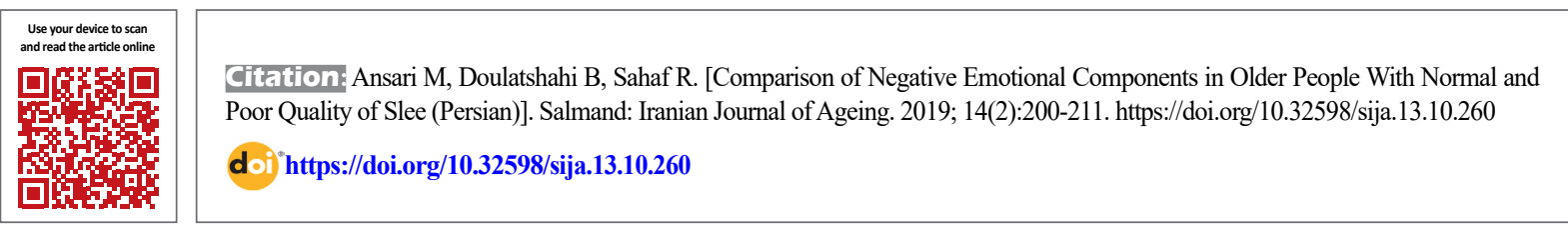

\section{(i) (3)}

Received: 01 Mar 2019 Accepted: 29 May 2019 Available Online: $01 \mathrm{Jul} 2019$

Key words: Sleep, Aged, Worry

\section{A B STRACT}

Objectives Sleep is one of the basic needs of every human being. Considering the factors affecting this area is of particular importance. The present study compares the negative emotional components, including rumination, worry, arousal anxiety in older people with sleep problems, and those with normal sleep.

Methods \& Materials This research is a causal-comparative study. The study population consisted of older people living in Tehran City in 2016. The study samples were selected by the convenience sampling method and based on inclusion and exclusion criteria. The inclusion criteria included being $\geq 60$ years old, lacking severe illnesses and disorders (whether physical and psychological). The exclusion criterion included the unwillingness to continue study at each stage. Of 200 older people participating in the study, 131 individuals were included in the study after group matching based on demographic variables. Pittsburgh sleep quality index, ruminative responses scale questionnaire, Pennsylvania state worry questionnaire, mood and anxiety symptom questionnaire, and anxious arousal scale were used to collect the study data. The reliability of the tools was also evaluated. The obtained data were analyzed by descriptive statistics of frequency, mean and by inferential statistics of the Chi-square, Man-Whitney $U$, and Independent t test in SPSS V. 18.

Results The Mean \pm SD age of the subjects was $68.5 \pm 6.78$ years, respectively. The mean scores of rumination were 54.039 in the elderly group with poor sleep quality and 44.919 in the group with normal sleep quality. Also, the mean scores of worry were 53.875 and 46.024 in the poor and normal sleep quality group, respectively. Finally, the mean arousal anxiety scores were 55.080 and 43.772 in the poor and normal sleep quality group, respectively. There was a statistically significant difference between the mean values of the two groups in terms of research variables $(P<0.001)$.

Conclusion According to the study results, rumination, worry, and arousal anxiety have a negative effect on the quality of sleep. Focusing on these negative emotional components as risk factors for the emergence and continuation of sleep disturbances can be useful in designing preventive interventions for sleep problems and reducing psychological disorders.

\section{* Corresponding Author:}

Behrouz Doulatshahi, PhD.

Address: Substance Abuse and Dependence Research Center, Department of clinical psychology, University of Social Welfare and Rehabilitation Sciences, Tehran, Iran.

Tel: +98 (21) 22180045

E-mail: dolatshahee@yahoo.com 


\section{Extended Abstract}

\section{Objectives}

A

ging is a natural process and of the stages of human growth. The improvements in living conditions and health care, as well as the longevity and life expectancy, have led to aging in societies $[1,2]$. With an increasing elderly population in Iran and the world, paying attention to this group's biopsychological health is of particular importance [3]. Aging is associated with quantitative and qualitative changes in the sleep pattern. Problems with falling asleep or sleep continuity, waking early in the morning and inability to fall back sleep are prevalent among the elderly [4]. Data are scarce on the negative emotional components in older people and their relation to their sleep quality; thus, this study aimed to compare the negative emotional components in older people with healthy and poor sleep quality.

\section{Methods \& Materials}

This was causal-comparative research. The study population consisted of all elderly residents of Tehran City, Iran, in $2016(\mathrm{n}=200)$. Of them, 200 samples were selected by visiting recreational, tourism, and sports centers based on the inclusion criteria and using convenience sampling technique. Inclusion criteria were $\geq 60$ years of age, ability to commu- nicate verbally and to read and write, cognitive health, no history of severe biopsychological disorders, no history of substance or alcohol or illegal medications abuse according to the self-reports, and providing informed consent forms to participate in the study. The exclusion criteria were the unwillingness to continue study participation.

For collecting data, a researcher-made demographic form was used in addition to the Pittsburgh Sleep Quality Index, Ruminative Responses Scale, Pennsylvania State Worry Questionnaire, and Mood and Anxiety Symptoms Questionnaire (Anxious Arousal Scale). If any of the study participants had difficulty with reading or understanding the questions, those were read and explained to them. Based on the cut-off point of 5 for sleep quality, the study participants were divided into two groups of healthy and poor sleep quality. After matching the subjects for demographic factors, 131 subjects participated in the study; 78 were assigned to the poor sleep quality group and 53 to the healthy sleep quality group. The collected data were analyzed in SPSS using descriptive statistics (frequency, mean, standard deviation) and statistical tests, including Chi-squared test, Mann-Whitney U test, ad Independent Samples t-test.

\section{Results}

Of 131 samples, $55 \%$ were male and $45 \%$ were female with the Mean $\pm \mathrm{SD}$ age of $68.5 \pm 6.78$ years. Moreover, $(80$ $90 \%$ ) of them were married living with the family; $(84 \%)$

Table 1. Comparing negative emotional components in the elderlies with poor and healthy sleep quality

\begin{tabular}{cccc}
\hline Variables & Group & Mean \pm SD & $\mathbf{n}$ \\
\hline \multirow{2}{*}{ Rumination } & Normal & $44.919 \pm 8.671$ & 53 \\
& Poor & $54.039 \pm 8.636$ & 78 \\
Worry & Normal & $46.024 \pm 9.291$ & 53 \\
& Poor & $53.875 \pm 8.842$ & 78 \\
\hline \multirow{2}{*}{ Anxious arousal } & Normal & $43.772 \pm 5.076$ & 53 \\
& Poor & $55.080 \pm 10.197$ & 78 \\
\hline
\end{tabular}

\begin{tabular}{|lccccc|}
\hline & \multicolumn{2}{c}{ Levene's Test } & \multicolumn{2}{c}{ t-test } \\
\cline { 2 - 6 } Variances Status & $\mathbf{F}$ & Sig. & $\mathbf{t}$ & $\mathbf{d f}$ & Sig. \\
\hline Equality of variances & 0.030 & 0.862 & -5.922 & 129 & 0.0001 \\
\hline Equality of variances & 0.092 & 0.762 & -4.887 & 129 & 0.0001 \\
\hline Inequality of variances & 26.637 & 0.0001 & -8.383 & 119.8 & 0.0001 \\
\hline
\end{tabular}


reported moderate economic status; $(50 \%)$ had a high school diploma and Bachelor's degree, and (31\%) reported elementary education. Chi-squared test results suggested no significant difference between the groups in terms of gender $\left(\mathrm{X}^{2}=0.002, \mathrm{P}=0.965\right)$, living conditions $\left(\mathrm{X}^{2}=0.146\right.$, $\mathrm{P}=0.702)$, and marital status $\left(\mathrm{X}^{2}=2.915, \mathrm{P}=0.088\right)$. MannWhitney $U$ test results revealed no significant difference between the groups concerning education (Mann-Whitney $\mathrm{U}$ test $=1904.500, \mathrm{Z}=-0.792, \mathrm{P}=0.429)$ and health status (Mann-Whitney U test $=1816.000, \mathrm{Z}=-1.501, \mathrm{P}=0.133$ ).

After matching the groups in terms of demographic characteristics, the study groups were compared with each other in terms of rumination, worry, and anxious arousal. For this purpose, the Independent Samples t-test was used (Table 1). Based on the Levene's test results, the assumption of equal variances was met for all variables, except for anxious arousal. There was a significant difference between the groups in terms of rumination, worry, and anxious arousal. This finding indicates that older people with poor sleep quality experienced significantly more rumination, worry, and anxious arousal.

\section{Conclusion}

Rumination, worry, and anxious arousal were significantly different in the elderlies with poor and healthy sleep quality. The obtained data suggested rumination and worry could predict sleep quality, which is consistent with previous studies [5-7]. Negative cognitive activities majorly affect sleep quality and its associated problems. This result was in agreement with those of Harvey [8]. A cognitive model presented by Harvey [9] suggested that sleep-related excessive worry and rumination cause arousal and emotional distress and attentional bias, leading to the development and maintenance of sleep disturbances. We found that rumination, worry, and anxious arousal negatively affected sleep quality. Focusing on these negative emotional components as the risk factors for the onset and continuation of sleep disorders can be useful in designing preventive interventions for sleep problems and reducing mental issues.

\section{Ethical Considerations}

\section{Compliance with ethical guidelines}

All ethical principles were considered in this article. The participants were informed about the purpose of the research and its implementation stages; they were also assured about the confidentiality of their information; Moreover, They were allowed to leave the study whenever they wish, and if desired, the results of the research would be available to them.
Funding

This research did not receive any specific grant from funding agencies in the public, commercial, or not-for-profit sectors.

Authors' contributions

All authors contributed in preparing this article.

Conflicts of interest

The authors declared no conflict of interest. 


\title{
مقايسه مؤلفههاى هيجانى منفى در افراد سالمند داراى كيفيت خواب طبيعى و ضعيف
}

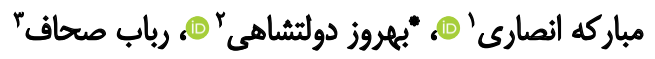

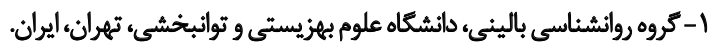

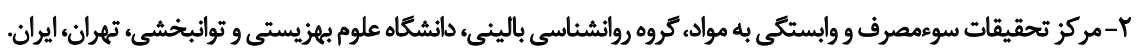

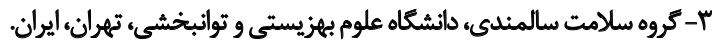

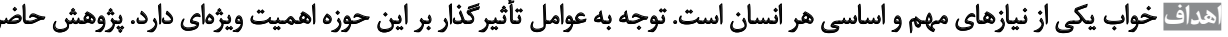

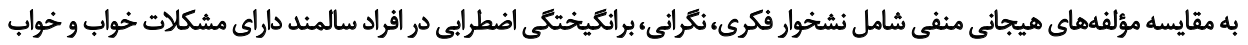

$$
\text { طبيعى يرداخته استئ. }
$$

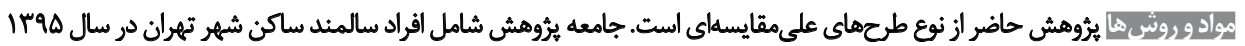

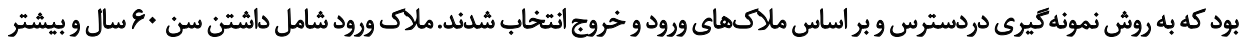

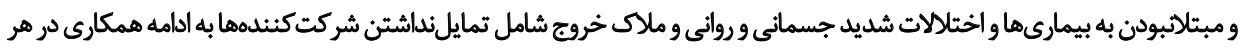

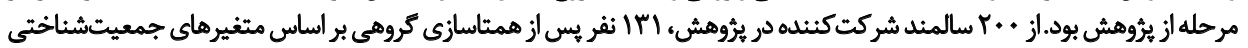

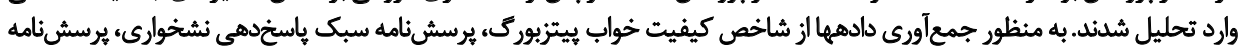

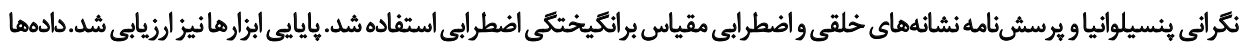

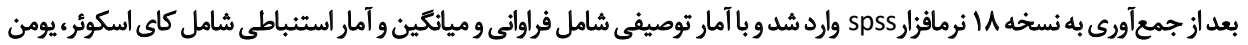

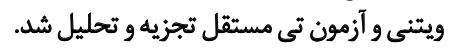

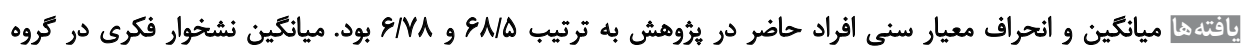

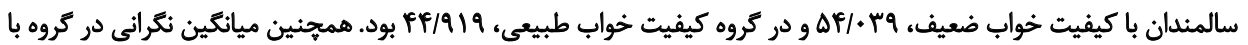

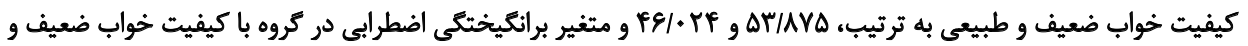

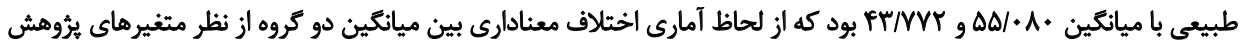

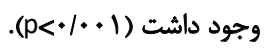

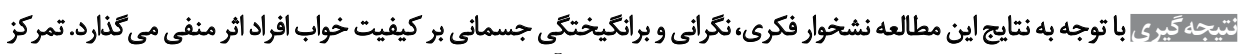

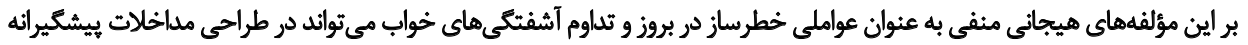

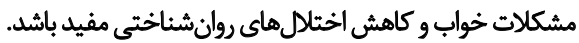

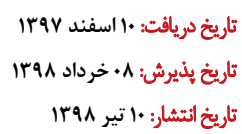

كليدوازٔهها:

خوابه سالخورده. اضطراب

به 1/T ميليارد نفر خواهد رسيد [r]. طبق آخرين آمار وزارت

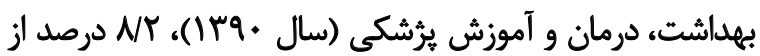

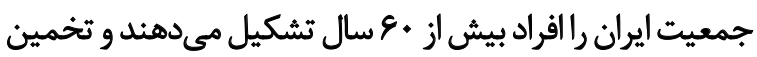

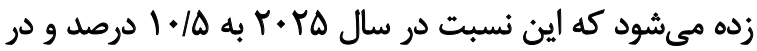

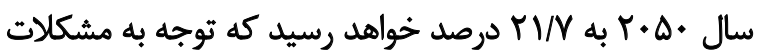

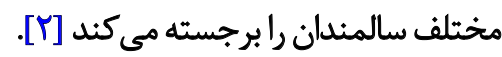

امروزه تعداد جمعيت سالمند بلهرعت رو به به افزايش است.

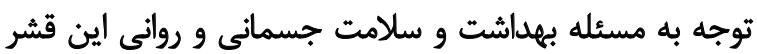

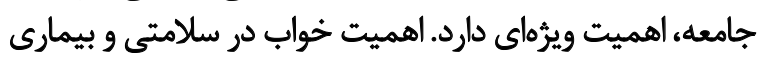

سالمندى فرايندى طبيعى و يكى از مراحل رشد و تكامل

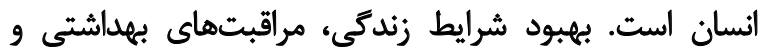

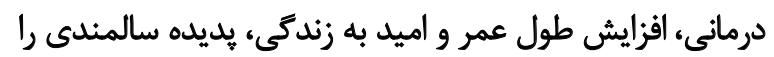

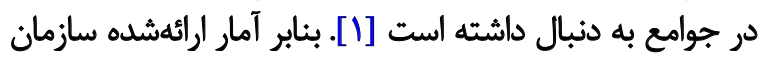

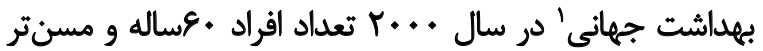

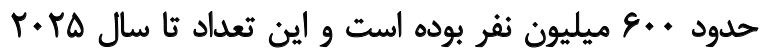

1. Th World Health Organization

مقدمه 
سببى در كاهش خوابشان ميدايند [•[]. با توجه به اينكه تجربه سالمندى در ايران آغاز شده است،

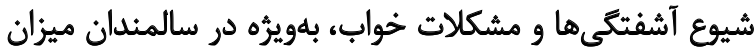

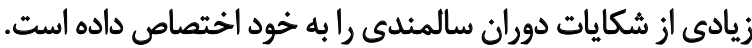

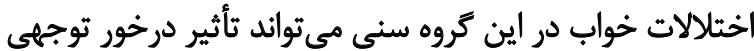

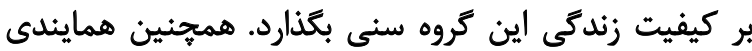

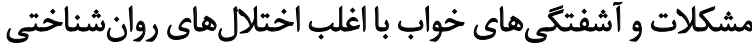

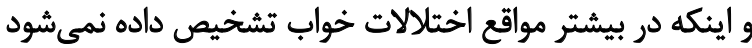

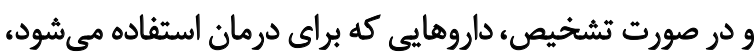
مى تواند تداخلات دارويى و عوارض جدائ دارئ ايجاد كند.

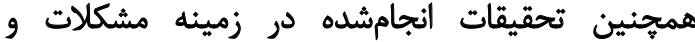

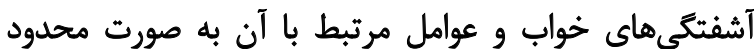

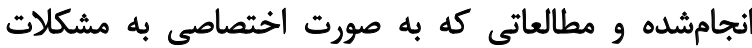

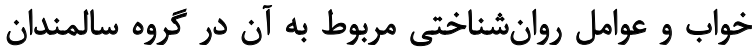

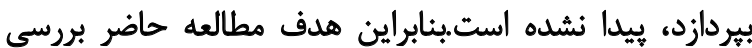

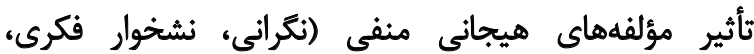

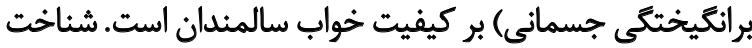

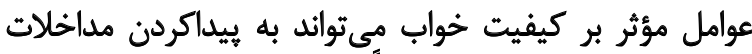

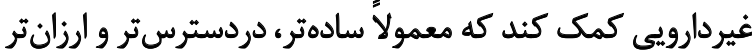

$$
\text { و كمعارضهتر از دارو است. }
$$

روش مطالعه

يثروهش حاضر از لحاظ هدف بنيادى كاربردى واز نوع طرحهاي

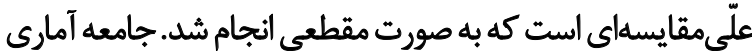

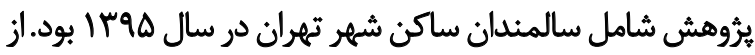

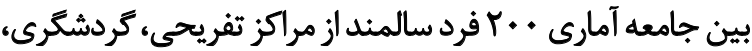

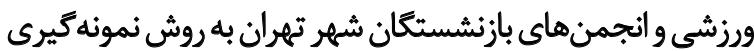

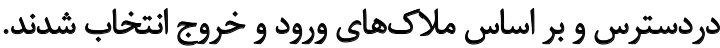

ع. ملاكهاي ورود به اين يُروهش عبارت بودند ازئ داشتن

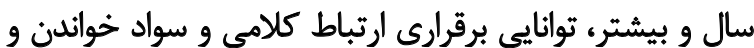

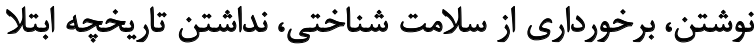

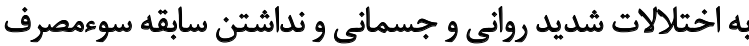

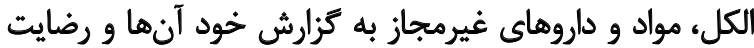

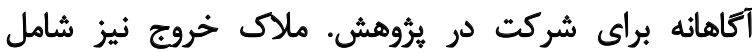

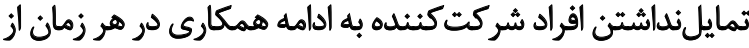

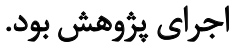

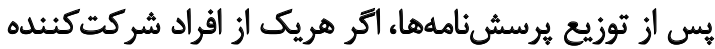

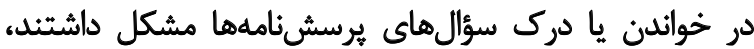

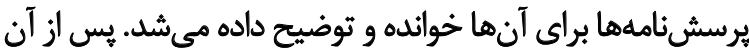

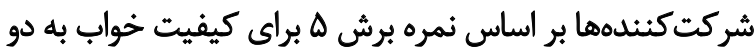

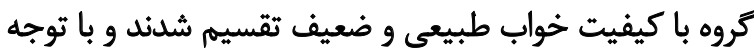

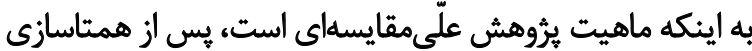

از زمان بقراط محل توجه بوده است وخواب آشفته، يك علت مهمه

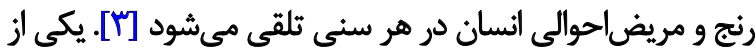

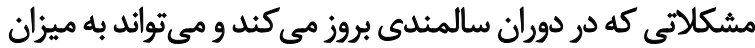

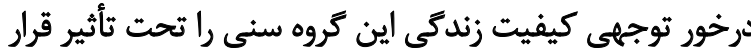

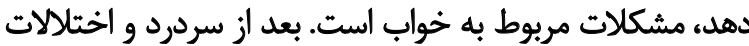

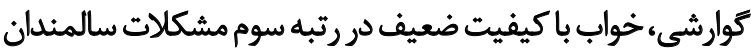

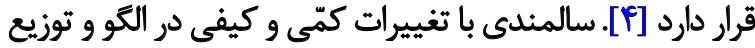

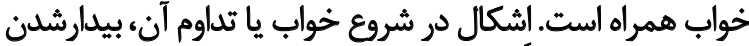

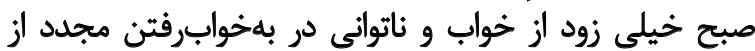

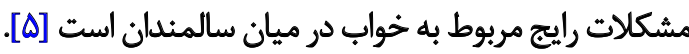

آشفتگى خواب در قالب الثوى آسيبذيذيرىاسترس تبيين

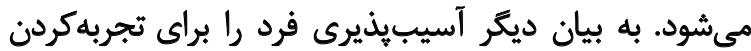

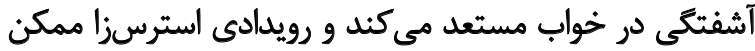

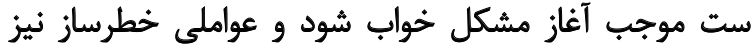

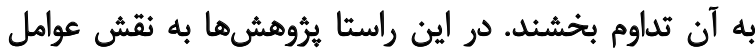

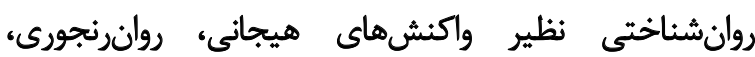

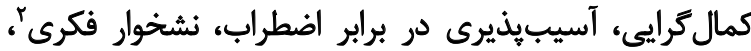

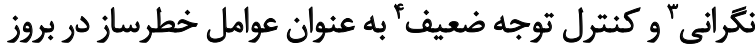

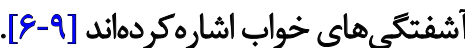

نكرانى، نوعى افكار تكرارشونده منفى و شامل زنجيرهاي از

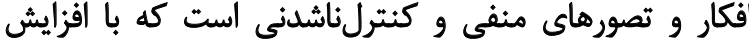

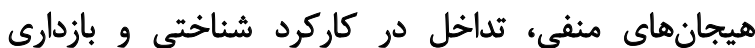

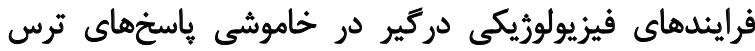

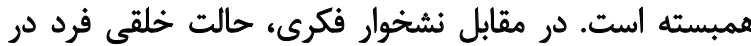

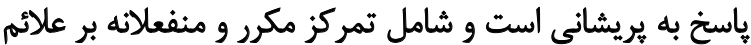

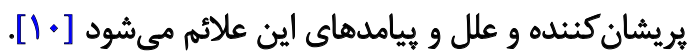

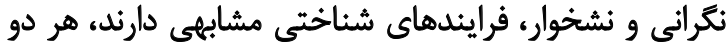

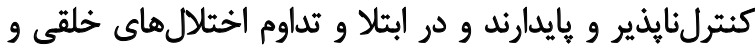

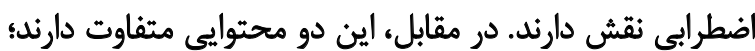

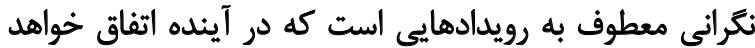

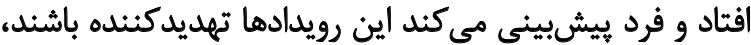

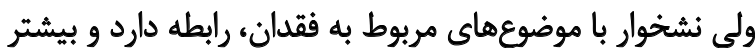

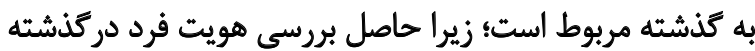

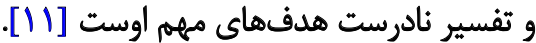

نكرانى و برانكيختكى جسمانى اضطراب، با تزارشهائ عينى

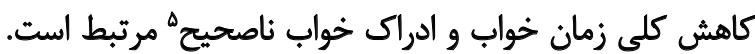

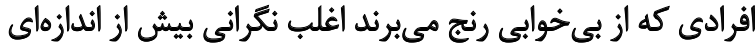

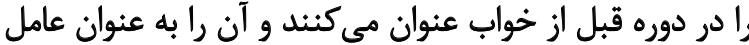

2. Rumination

3. Worry

4. Poor attentional control

5. Inaccurate 


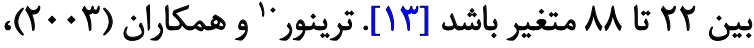

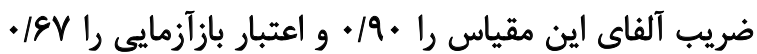

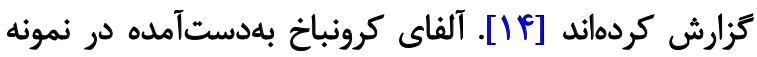

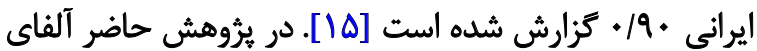

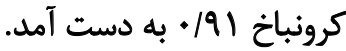

\section{برسش ثامهنتكر انى ئنسيلوانيا"}

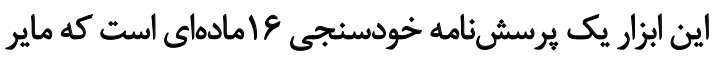

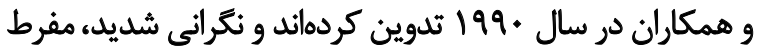

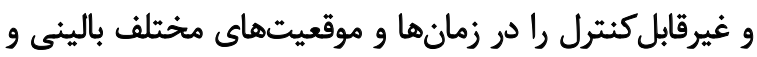

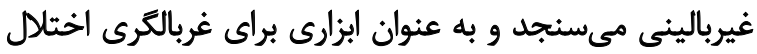

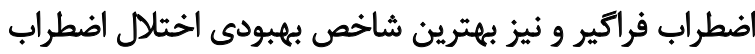

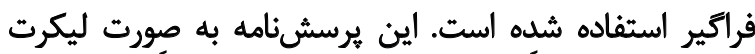

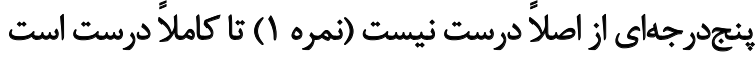

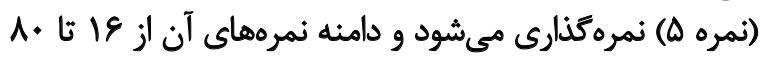
متغير است كه نمره بيشتر نشانكر اضطراب بالاتر است.

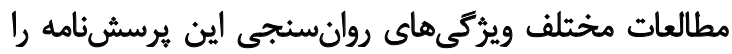

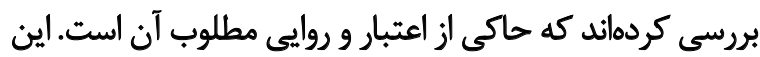

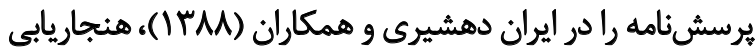

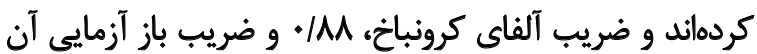

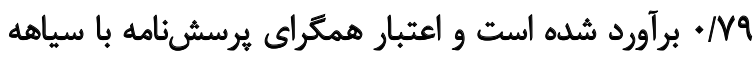

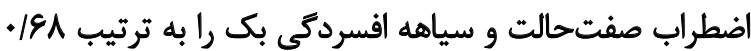

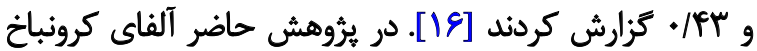

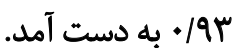

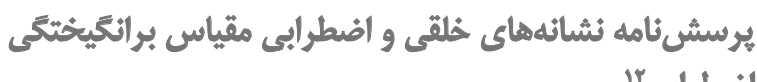
اضطرابى

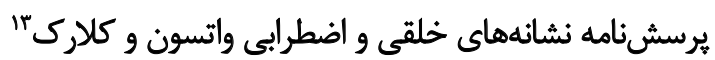

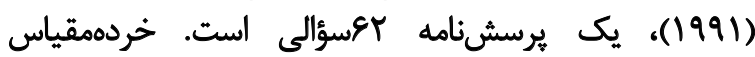

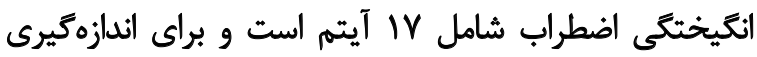

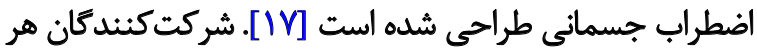

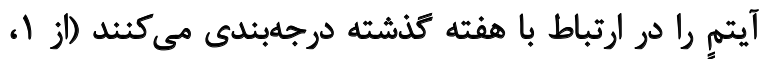

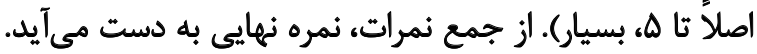

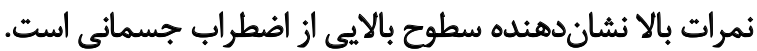

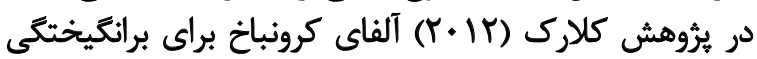

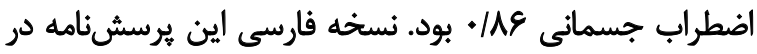

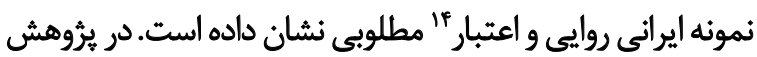

10. Treynor

11. Pennsylvania State Worry Questionnaire (PSWQ)

12. Mood and Anxiety Symptom Questionnaire Anxious Arousal Scale (MASQ-ANX)

13. Watson \& Clark

14. Reliability

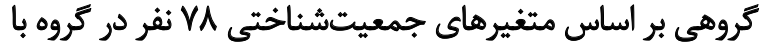

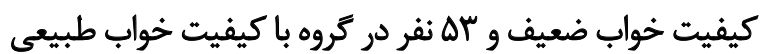

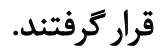

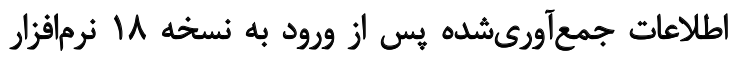

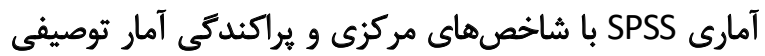

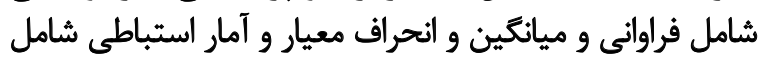

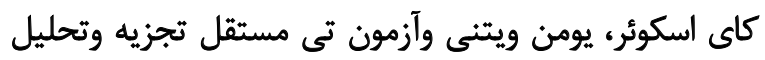

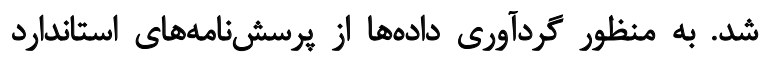

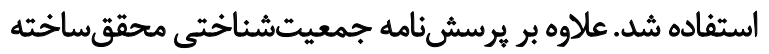

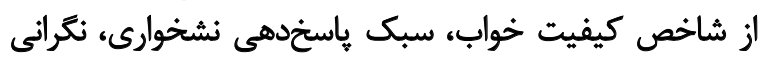

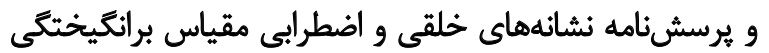

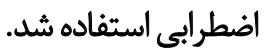

\section{شاحُص كيفيث حُواب يبيتزبورَّل}

بايسى و و همكارانش (1919) اين مقياس را براى ارزيابى

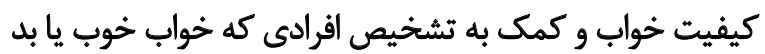

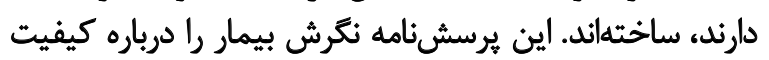

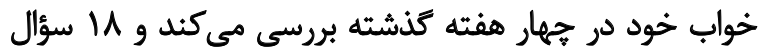

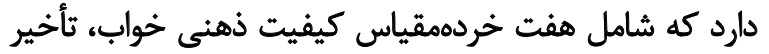

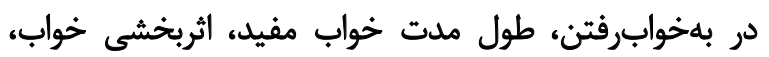

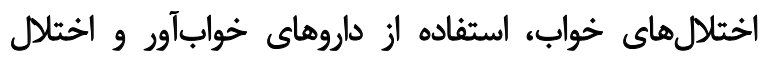

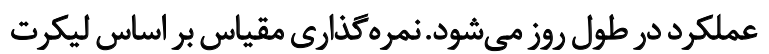

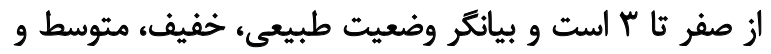

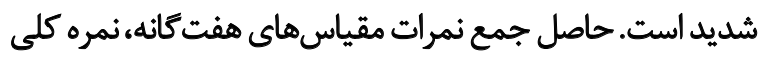

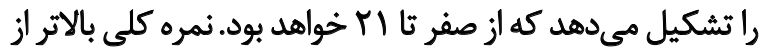

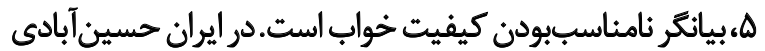

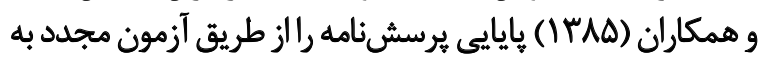

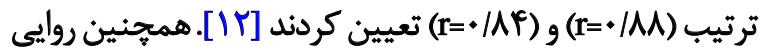

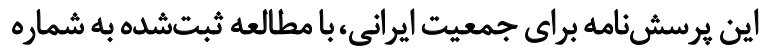
حاضر خrr.

حاضر ضريب آلفاى كرونباخ •/N/ • محاسبه.

يرسش نامه سبك ياسنْدهى نشخوارى

مقياس سبك باسخ نشخوارى، زيرمقياسى از يرسشنامه

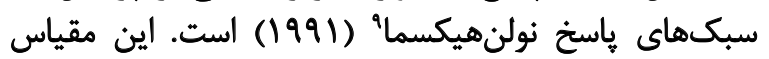

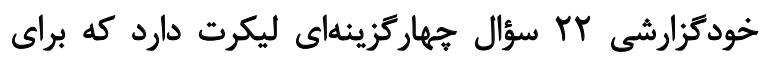

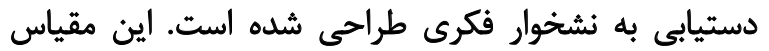

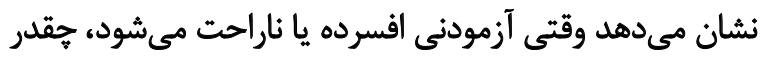

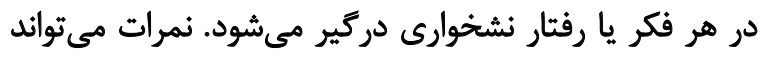

\footnotetext{
6. Pittsburgh Sleep Quality Index (PSQI)

7. Buysse

8. Ruminative Responses Scale (RRS)

9. Nolen-hoeksema
} 
بين دو كروه سالمندان با كيفيت خواب طبيعى و خواب ضعيف

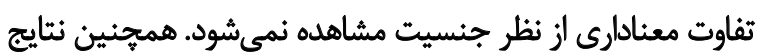

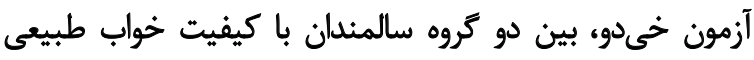

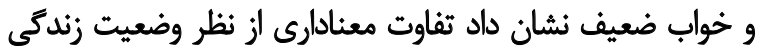

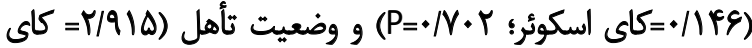

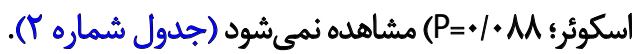

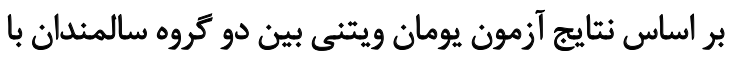

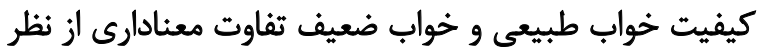

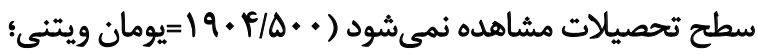

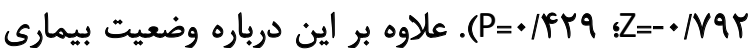

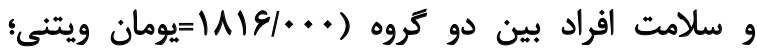

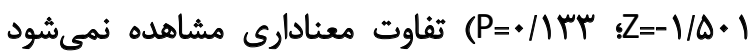

$$
\text { (جدول شماره س). }
$$

تحليل نتايج آزمون خىدو، بين دو كروه سالمندان با كيفيت

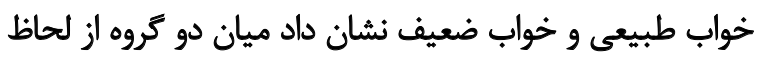

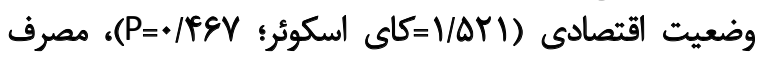

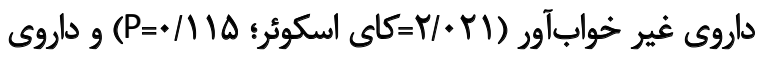

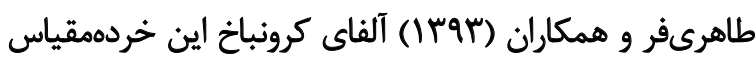

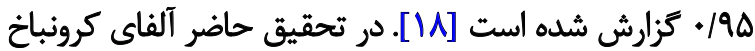

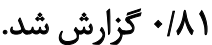

يافتهها

نمونه يُوهش حاضر شامل آس ا فرد سالمند ساكن شهر تهران

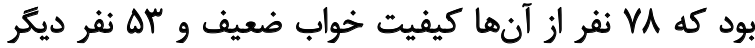

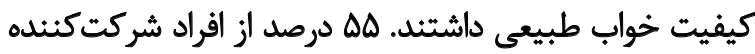

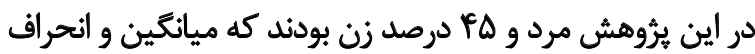

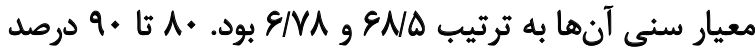

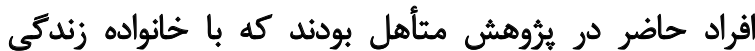

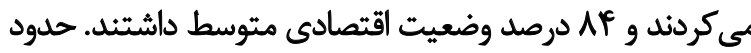

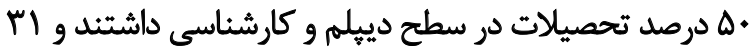

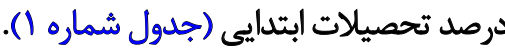

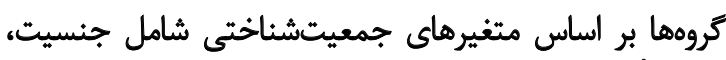

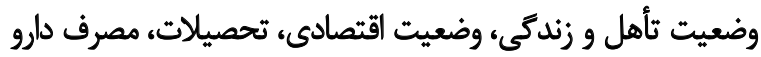

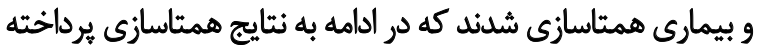

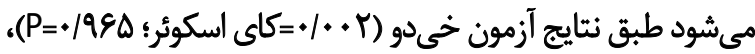

جدول ا. اطلاعات جمعيتشئاختى افراد سالميد

\begin{tabular}{|c|c|c|c|}
\hline درصد & تعداد & & \\
\hline Pa & $\Delta q$ & زن & \multirow{3}{*}{ جنسيت } \\
\hline$\Delta \Delta$ & $r$ & مرد & \\
\hline $1 .$. & $|r|$ & جمع & \\
\hline$A \backslash / N$ & $1 \cdot V$ & 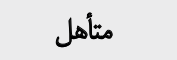 & \multirow{3}{*}{ وضعيت تأهل } \\
\hline$W^{N}$ & tf & همسر فوت شده & \\
\hline $1 .$. & $|r|$ & جمع & \\
\hline $1 Q / \pi$ & $11 \mathrm{~V}$ & با خانواده & \multirow{3}{*}{ 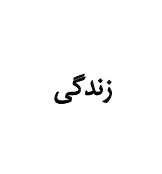 } \\
\hline $1.1 V$ & if & تنه & \\
\hline $1 \ldots$ & $|m|$ & جمع & \\
\hline$r \mid r$ & fi & ابتدايى & \multirow{6}{*}{ ميزان تحصيلات } \\
\hline $1 . N$ & if & سيكل & \\
\hline$r \cdot 10$ & $r$ & دييلم & \\
\hline$\pi / N$ & m & ليسائس & \\
\hline$r / \Lambda$ & $\Delta$ & فوقليسائس & \\
\hline $1 \ldots$ & $|r|$ & جمع & \\
\hline$V / s$ & 1. & ضعيف & \multirow{4}{*}{ وضعيت اقتمصادى } \\
\hline Af & 11. & متوسط & \\
\hline$N F$ & 11 & ب̣ & \\
\hline 1. & $|r|$ & جمع & \\
\hline
\end{tabular}


جدول r. نتايج همتاسازى مربوط به جنسيت، وضعيت تأهل و زندكى شركت كنثدكان با كيفيت خواب طبيعى و ضعيف

\begin{tabular}{|c|c|c|c|c|c|c|c|c|c|c|c|}
\hline معنادارى & كالسكوثر & درصد & فراواتى & زندكى & درصد & فراوانى & وضعيت ثأهل & درصد & فراوانى & جثسيت & كرو \\
\hline \multirow{6}{*}{$\begin{array}{l}+/ Q S \Delta \\
+/ * M \\
+N \cdot Y\end{array}$} & \multirow{6}{*}{$\begin{array}{l}+/ .+r \\
r / 910 \\
. / 148\end{array}$} & $9 / 4$ & $\Delta$ & تنها & $M V$ & $\mathrm{pV}$ & متأهل & $r \Delta / r$ & me & زن & \multirow{3}{*}{ كيفيت خواب } \\
\hline & & 9.18 & \&A & با خانواده & $11 / r$ & 8 & همسر فوت شده & $\Delta F / V$ & rq & مرد & \\
\hline & & $1+$. & بr & جمع كل & $1+$. & $\Delta r$ & جمع كل & $1 \ldots$ & "H & جمع كل & \\
\hline & & $11 / 0$ & 9 & تنهيا & $V g / q$ & q. & متأهل & $p e / q$ & ra & زن & \multirow{3}{*}{ كيفيت خوابف } \\
\hline & & WD & 99 & با خانواده & $m / l$ & M & همسر فوت شده & $\Delta \Delta / 1$ & r & مرد & \\
\hline & & 1.0 & $\mathrm{~V} A$ & جمع كل & $1+$. & $\mathrm{V} A$ & جمع كل & $1 \ldots$ & $v \wedge$ & جمع كل & \\
\hline
\end{tabular}

닌

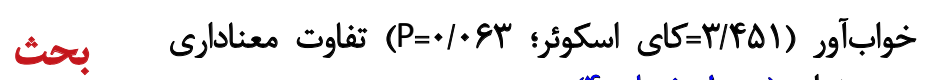
وجود ندارد (جدول شماره f).

هدف يُرؤش حاضر بررسى تأثير مؤلفههاي هيجانى منفى

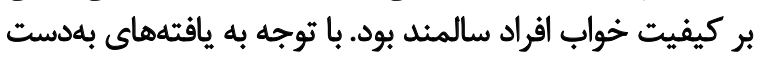

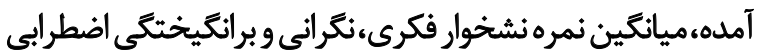

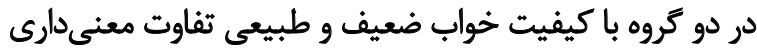

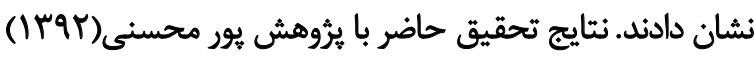

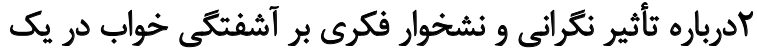

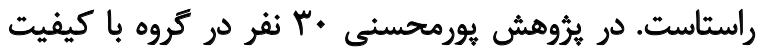

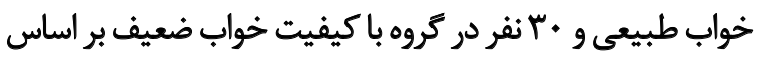

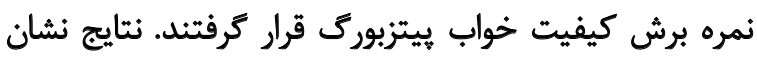

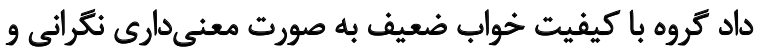

نشخوار فكرى بيشترى راتجربه مي كنيند [11]

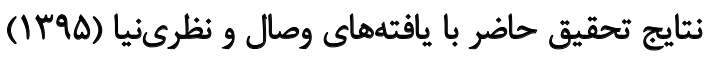

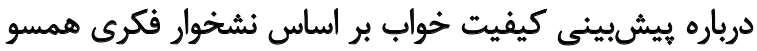

يس از همثاسازى دو كَروه بر اساس متغيرهاي جمعيتشيناختى

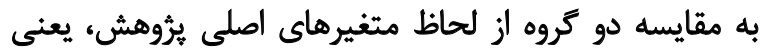

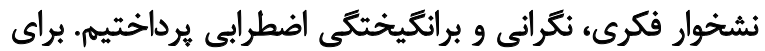

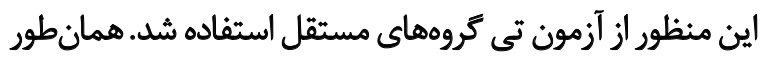

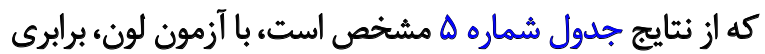

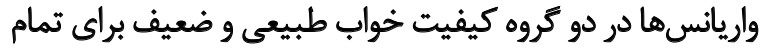

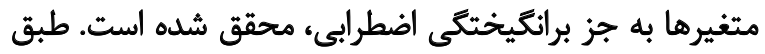

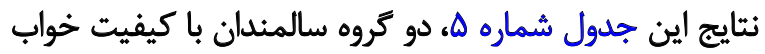
طبيعى و ضعيف در تمامى متغيرها (نشخوار فكرى، نكراني، داني،

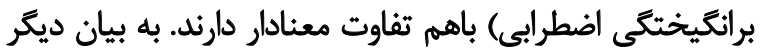

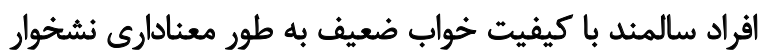

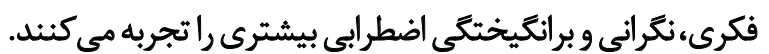

جدول r . نتايج همثاسازى مربوط به سطح تحصيلات و وضعيت بيمارى افراد با كيفيت خواب طبيعي و ضعيف

\begin{tabular}{|c|c|c|c|c|c|c|c|c|c|}
\hline معنادارى & $\mathbf{Z}$ & يومن ويتثى & درصد & فراوانى & بيمارى & so & فراوانى & تحصيلات & تروه \\
\hline & & & $r q / r$ & rr & نبود بيمارى & $r+/ r$ & is & ابتلاييى & \multirow{5}{*}{ كيفيت خواب طبيعى } \\
\hline & & & $1 \pi / r$ & $\checkmark$ & فشار خون & $11 / \mu$ & 8 & سيكل & \\
\hline & & & $r / \Lambda$ & r & قتند خُون & $r F / \Delta$ & ir & دييلم & \\
\hline & & & • & • & درد مزمن & TNM & 10 & ليسائس & \\
\hline & & & $r / A$ & r & قلبي & $\Delta / V$ & $r$ & فوق ليسائس & \\
\hline - perq &.$- / M q Y$ & $19.7 / 0 .$. & $1 \cdots$ & هr & جمع كل & $1 .$. & هM & جمع كل & \\
\hline \multirow[t]{6}{*}{ זיזות. } & $1 / 0.1$ & $|A| \& \mid \ldots$ & $9 / 9$ & سه & نبود بيمارى & $\pi / 1$ & ro & ابتلايي & \multirow{6}{*}{ كيفيت خواب ضعيف } \\
\hline & & & $10 / \uparrow$ & ir & فشار خون & $1 . / r$ & $\wedge$ & 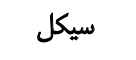 & \\
\hline & & & 9 & $\checkmark$ & قند خون & $m e / s$ & iv & دييلم & \\
\hline & & & $r / \Lambda$ & r & درد مزمن & $r \cdot / \Delta$ & is & ليسائس & \\
\hline & & & $r / A$ & r & قلبيى & r/S & r & فوق ليسانس & \\
\hline & & & $1 \ldots$ & va & جمع كل & $1 .$. & va & جمع كل & \\
\hline
\end{tabular}

ك 
جدول F. نتايج همتاسازى مربوط به وضعيت اقتصادى و مصرف داروى افراد با كيفيت خواب طبيعى و ضعيف

\begin{tabular}{|c|c|c|c|c|c|c|c|c|c|c|c|}
\hline معنادارى & كاسكوئر & درصد & فراوانى & خواب أور & درصد & فراوانى & غيرخوابآور & درصد & فراوانى & اقتّاديت & تموه \\
\hline \multirow{10}{*}{$\begin{array}{l}. / 18 \mathrm{P} \\
. / 110 \\
.1 .84\end{array}$} & \multirow{10}{*}{$\begin{array}{l}\text { VATI } \\
Y / \cdot Y I \\
r / F \Delta I\end{array}$} & \multirow[b]{2}{*}{$9+18$} & & \multirow[b]{2}{*}{ ن ندارد } & \multirow[b]{2}{*}{ va/r } & \multirow[b]{2}{*}{ ft } & \multirow[b]{2}{*}{ نلثارد } & $11 / \pi$ & 8 & بالا & \multirow{5}{*}{ خيفيت } \\
\hline & & & & & & & & $r a / r$ & Pr & متوسط & \\
\hline & & $9 / 4$ & $\Delta$ & دارد & $r+/ A$ & 11 & دارد & & & & \\
\hline & & \multirow[t]{2}{*}{$1 .}$. & \multirow[t]{2}{*}{$\Delta \mu^{\mu}$} & \multirow{2}{*}{ جمع كل } & \multirow[t]{2}{*}{$1 \ldots$} & \multirow[t]{2}{*}{$\Delta \mu$} & \multirow{2}{*}{ جمع كل } & $9 / 1^{6}$ & $\Delta$ & ضعيف & \\
\hline & & & & & & & & $1 .$. & $\Delta \mu^{\mu}$ & جمع كل & \\
\hline & & \multirow{2}{*}{ VNY } & \multirow[b]{2}{*}{81} & \multirow[b]{2}{*}{ ندارد } & \multirow{2}{*}{$9 V / 9$} & \multirow{2}{*}{ "L } & \multirow[b]{2}{*}{ ندارد } & $s / f$ & $\Delta$ & بالا & \multirow{5}{*}{ ضيفيت } \\
\hline & & & & & & & & YNY & \&A & مثّوسط & \\
\hline & & $r / / \Lambda$ & IV & دارد & $M T / 1$ & ro & دارد & & & & \\
\hline & & \multirow[t]{2}{*}{$1+}$. & \multirow[t]{2}{*}{$\mathrm{V} A$} & \multirow{2}{*}{ جمع كل } & \multirow[t]{2}{*}{$1 \ldots$} & \multirow[t]{2}{*}{$v i$} & \multirow[t]{2}{*}{ جمع كل } & $1 \pi$ & $\omega$ & صعيق & \\
\hline & & & & & & & & 10. & $\mathrm{VA}$ & جمع كل & \\
\hline
\end{tabular}

Lب

جدول ه. مقايسه ميانكين مؤلفههاى هيجاني منفي در دو كروه سالمندان با كيفيت خواب طبيعى و ضعيف

\begin{tabular}{|c|c|c|c|c|c|c|c|c|c|}
\hline \multicolumn{3}{|c|}{ آزمون تى } & \multicolumn{2}{|c|}{ آزمون لون } & \multirow{2}{*}{ متغيرها } & \multirow{2}{*}{ فراوانى } & \multirow{2}{*}{ ميانكين+|نحراف معيار } & \multirow{2}{*}{ كروه } & \multirow{2}{*}{ متغيرها } \\
\hline معنادارى & df & $\mathbf{t}$ & معنادارى & $\mathbf{F}$ & & & & & \\
\hline \multirow{2}{*}{$.1+\ldots 1$} & \multirow{2}{*}{ irq } & \multirow{2}{*}{-Q/ATr } & \multirow{2}{*}{. INET } & \multirow{2}{*}{.$/ \cdot r$} & برابرى & $\Delta r$ & & خواب طبيعى & نشخولار \\
\hline & & & & & والريانسها & vi & $\Delta H / H \pm V / R T$ & خواب ضعيف & فكرى \\
\hline \multirow{2}{*}{$.1+\ldots+1$} & \multirow{2}{*}{179} & \multirow{2}{*}{$-F / M Y$} & \multirow{2}{*}{. NET } & \multirow{2}{*}{.1 .94} & برابرى & $\Delta r^{r}$ & eq/retq/rq & خواب طبيعى & \multirow{2}{*}{ نكراني } \\
\hline & & & & & واريانسها & $\mathrm{va}$ & QH/AVINAF & خواب ضعيف & \\
\hline \multirow{2}{*}{$+1+\ldots 1$} & \multirow{2}{*}{$119 / 1$} & \multirow{2}{*}{-N N } & \multirow{2}{*}{$+1+.+1$} & \multirow{2}{*}{ reletr } & يرابرثبودن & $\Delta r$ & $P r / W \pm \Delta / V^{2}$ & خواب طبيعى & براتكيختكي \\
\hline & & & & & واريانسها & va & $\Delta \Delta / \cdot \Delta \pm 1 \cdot / 19$ & خواب ضعيف & اضطرابى \\
\hline
\end{tabular}

乩

سبب بروز برانكيختكى و آشفتگى هاى هيجانى شده و سوكيرى

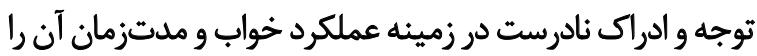

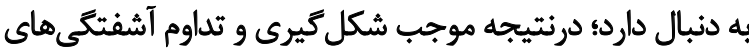

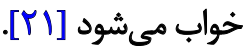

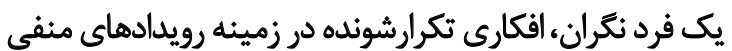

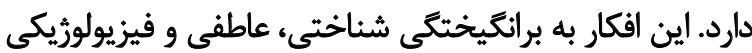

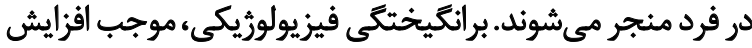

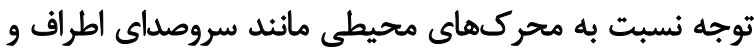

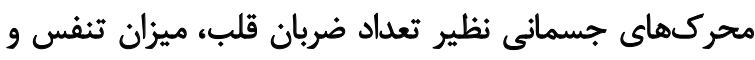

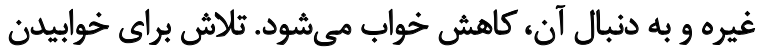

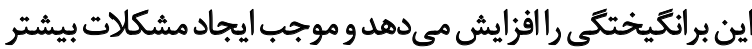

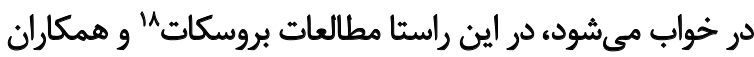

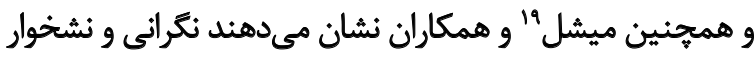

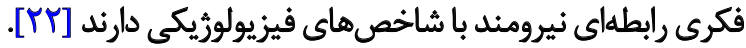

18. Brosschot

19. Mitchell

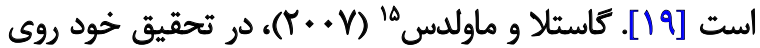

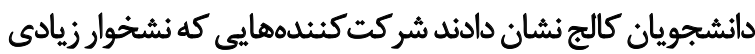

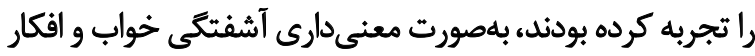

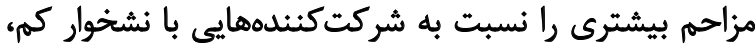

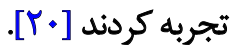

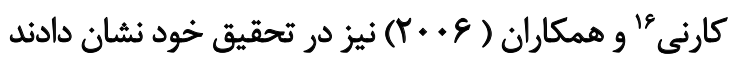

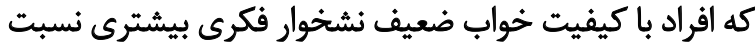

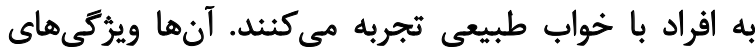

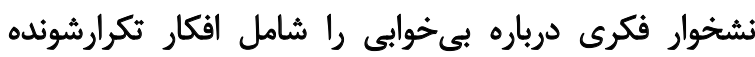

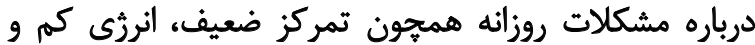

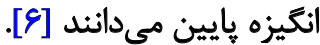

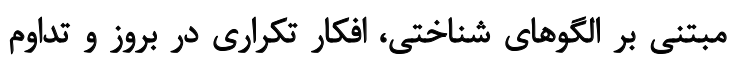

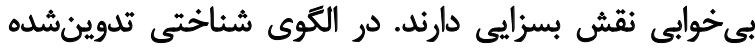

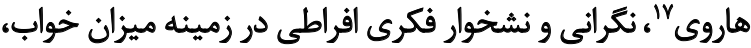

15. Guastella \& Moulds

16. Carney

17. Harvey 


\section{تعارض مئاقع}

بنابر اظهار نويسندكان، اين مقاله تعارض منافع ندارد.

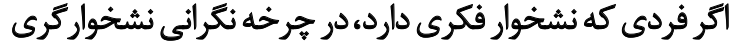

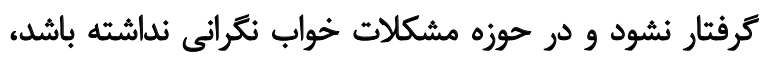

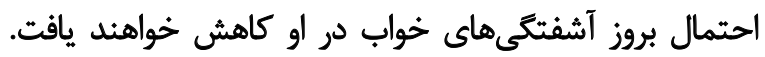

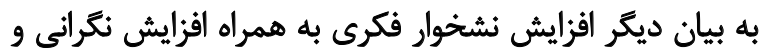

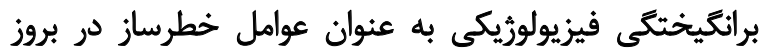
آشفتگى هاى خواب به شمار مئرودي نتيجه كيرى نهايى

با توجه به آنجه كفته شد، افكار تكرارشونده منفى به شكل

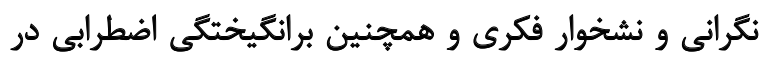

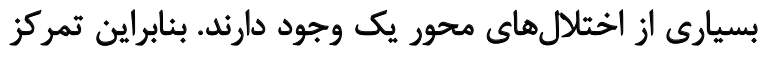

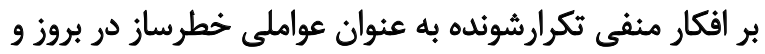

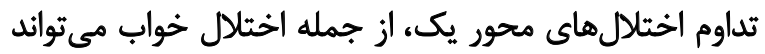

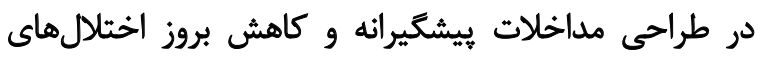
روانشناختى، مفيد باشد.

از محدوديتهاى اين يُووهش مي توان به مقطعىبودين

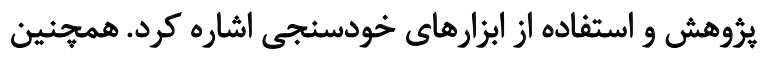

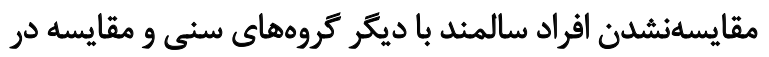

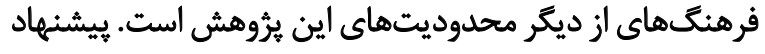

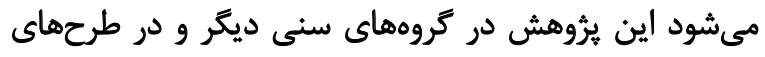

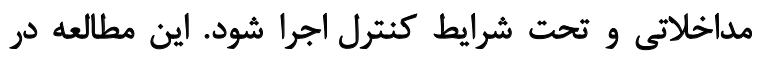

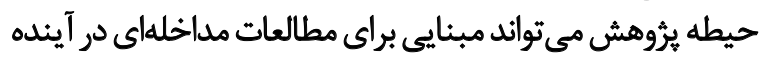

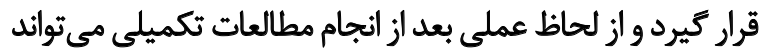

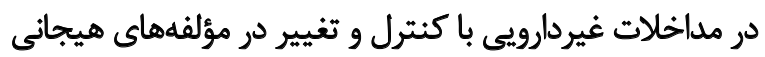
منفى، در بيشكيرى و درمان اختلالات خواب استفاده شود. ماحظات اخلاقي

$$
\text { يبروى از اصول الخلاق يثوهش }
$$

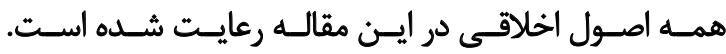

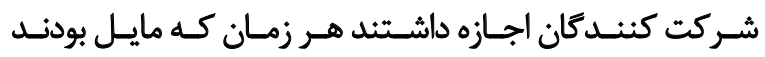

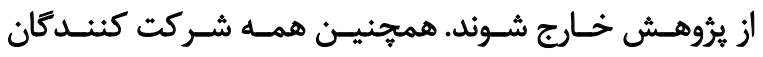

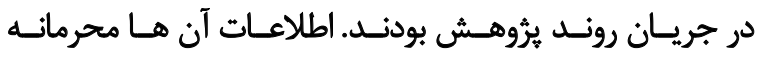

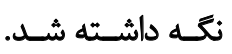

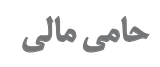

اين يُروهش از هيج اركان يا نهاد حمايتى هيجّونه حمايت مالى دريافت نكرده است. هشاركت نويسندكان تمام نويسندكان در آمادمسازى اين مقاله مشاركت داشتهاند. 


\section{References}

[1] Bekhet A, Zauszniewski J. Mental health of elders in retirement communities: Is loneliness a key factor? Archives of Psychiatric Nursing. 2012; 26(3):214-24. [DOI:10.1016/j.apnu.2011.09.007] [PMID] [PMCID]

[2] Ansari M, Doulatshahi B, Sahaf R. Comparison of negative emotional components in older people with normal and poor quality of sleep. Iranian Journal of Ageing. Forthcoming. [DOI: 10.32598/ sija.13.10.260]

[3] Afkham Ebrahimi A, Ghale Bandi, M, Salehi, M, Kafian Tafti, A, Vakili, Y, Akhlaghi Farsi, E. [Sleep parameters and the factors affecting the quality of sleep in patients attending selected clinics of Rasoul-e-Akram Hospital (Persian)]. Razi Journal of Medical Sciences. 2008; 15(1):31-8

[4] Seyyedrasouli A, Valizadeh L, Nasiri Kh, Zamandadeh V, Goljarian S, Ghasemi A. [The effect of therapeutic reflection on the elderly sleep quality (Persian)]. Quarterly Journal of Nursing, Surgery. 1392; 2(1):11-8.

[5] Allah ES, Abdel-Aziz HR, El-Seoud AR. Insomnia: Prevalence, risk factors, and its effect on quality of life among elderly in Zagazig City, Egypt. Journal of Nursing Education and Practice. 2014; 4(8):52-69. [DOI:10.5430/jnep.v4n8p52]

[6] Carney CE, Edinger JD, Meyer B, Lindman L, Istre T. Symptomfocused rumination and sleep disturbance. Behavioral Sleep Medicine. 2006; 4(4):228-41. [DOI:10.1207/s15402010bsm0404_3] [PMID]

[7] Fernández-Mendoza J, Vela-Bueno A, Vgontzas AN, RamosPlatón MJ, Olavarrieta-Bernardino S, Bixler EO, et al. Cognitiveemotional hyperarousal as a premorbid characteristic of individuals vulnerable to insomnia. Psychosomatic Medicine. 2010; 72(4):397-403. [DOI:10.1097/PSY.0b013e3181d75319] [PMID]

[8] Gosling JA, Batterham PJ, Christensen H. Cognitive-behavioural factors that predict sleep disturbance 4 years later. Journal of Psychosomatic Research. 2012; 73(6):424-9. [DOI:10.1016/j.jpsychores.2012.08.011] [PMID]

[9] van de Laar M, Verbeek I, Pevernagie D, Aldenkamp A, Overeem S. The role of personality traits in insomnia. Sleep Medicine Reviews. 2010; 14(1):61-8. [DOI:10.1016/j.smrv.2009.07.007] [PMID]

[10] Mitchell L, Mogg K, Mogg BP. Relationships between insomnia, negative emotionality and attention control. Sleep and Biological Rhythms. 2012; 10(3):237-43. [DOI:10.1111/j.14798425.2012.00567.x]

[11] Pourmohseni F. [Effect of rumination and anxiety in sleep disturbances (Persian)]. Daneshvar Medicine Journal. 1392; 21(108):1-12

[12] Hosseinabadi R, Tabrizi KN, Esmaiel ZP, Karimloo.M, Maddah SS. [The effects of Pressure massage on geriatrics sleep quality (Persian)]. Rehabilitation. 2008; 9(2):8-14.

[13] Nolen-Hoeksema S, Morrow J. A prospective study of depression and posttraumatic stress symptoms after a natural disaster: The 1989 Loma Prieta earthquake. Journal of Personality and Social Psychology. 1991; 61(1):115-21. [DOI:10.1037/00223514.61.1.115] [PMID]

[14] Treynor W, Gonzalez R, Nolen-Hoeksema S. Rumination reconsidered: A psychometric analysis. Cognitive Therapy and Research. 2003; 27(3):247-59.
[15] Darghahian R, Mohammadkhani Sh, Hosni C, Shams J. [The effect of metacognitive therapy on improving metacognitive beliefs, rumination and depression symptoms (Persian)]. Quarterly Journal of Clinical Psychology. 2011; 1(3):81-103.

[16] Dehshiri GR, Golzari M, Borjali A, Sohrabi F. [Psychometrics particularity of farsi version of Pennsylvania state worry questionnaire for college students (Persian)]. Journal of Clinical Psycology. 2009; 1(4):67-75.

[17] Watson D, Clark LA. Mood and anxiety symptom questionnaire. Iowa: University of Iowa; 1991. [DOI:10.1037/t13679-000]

[18] Taherifar Z, Ferdowsi S, Motabi F, Mazaheri M, Fata L. [The role of mediating deficits in emotion regulation strategies in the relationship between negative emotional excitement and immune motivation with general anxiety symptoms (Persian)]. Two Quarterly Iranian Psychological Association. 2016; 10(2):51-66.

[19] Vesal M, Nazarinia M. [Prediction of depression and sleep quality based on thought rumination and its components (inhibition and reflection) in patients with rheumatoid arthritis (Persian)]. Andishe va Raftar. 2016; 11(41):47-56

[20] Guastella AJ, Moulds ML. The impact of rumination on sleep quality following a stressful life event. Personality and Individual Differences. 2007; 42(6):1151-62. [DOI:10.1016/j.paid.2006.04.028]

[21] Harvey AG. A cognitive model of insomnia. Behaviour Research and Therapy. 2002; 40(8):869-93. [DOI:10.1016/S00057967(01)00061-4]

[22] Brosschot JF, Gerin W, Thayer JF. The perseverative cognition hypothesis: A review of worry, prolonged stress-related physiological activation, and health. Journal of Psychosomatic Research 2006; 60(2):113-24. [DOI:10.1016/j.jpsychores.2005.06.074] [PMID] 
This Page Intentionally Left Blank 